\title{
Comparison of the Mismatch Repair System between Primary and Metastatic Colorectal Cancers Using Immunohistochemistry
}

Jiyoon Jung · Youngjin Kang

Yoo Jin Lee · Eojin Kim

Bokyung Ahn · Eunjung Lee

Joo Young Kim · Jeong Hyeon Lee

Youngseok Lee · Chul Hwan Kim

Yang-Seok Chae

Department of Pathology, Korea University Anam Hospital, Seoul, Korea

Received: September 2, 2016

Revised: November 24, 2016

Accepted: December 9, 2016

Corresponding Author

Yang-Seok Chae, MD

Department of Pathology, Korea University Anam

Hospital, 73 Inchon-ro, Seongbuk-gu, Seoul 02841,

Korea

Tel: $+82-2-920-5595$

Fax: $+82-2-920-6576$

E-mail: chaeys21@korea.ac.kr
Background: Colorectal cancer (CRC) is one of the most common malignancies worldwide. Approximately $10 \%-15 \%$ of the CRC cases have defective DNA mismatch repair (MMR) genes. Although the high level of microsatellite instability status is a predictor of favorable outcome in primary CRC, little is known about its frequency and importance in secondary CRC. Immunohistochemical staining ( $\mathrm{IHC}$ ) for MMR proteins (e.g., MLH1, MSH2, MSH6, and PMS2) has emerged as a useful technique to complement polymerase chain reaction (PCR) analyses. Methods: In this study, comparison between the MMR system of primary CRCs and paired liver and lung metastatic lesions was done using $\mathrm{IHC}$ and the correlation with clinical outcomes was also examined. Results: Based on IHC, 7/61 primary tumors (11.4\%) showed deficient MMR systems, while $13 / 61$ secondary tumors (21.3\%) showed deficiencies. In total, 44 cases showed proficient expression in both the primary and metastatic lesions. Three cases showed deficiencies in both the primary and paired metastatic lesions. In 10 cases, proficient expression was found only in the primary lesions, and not in the corresponding metastatic lesions. In four cases, proficient expression was detected in the secondary tumor, but not in the primary tumor. Conclusions: Although each $\mathrm{IHC}$ result and the likely defective genes were not exactly matched between the primary and the metastatic tumors, identical results for primary and metastatic lesions were obtained in $77 \%$ of the cases (47/61). These data are in agreement with the previous microsatellite detection studies that used PCR and IHC.

Key Words: Colorectal neoplasms; DNA mismatch repair; Microsatellite instability; Immunohistochemistry
Approximately $10 \%-15 \%$ of the colorectal cancer (CRC) cases have a defective DNA mismatch repair (MMR) gene. ${ }^{1}$ As a result of these defects, microsatellites are predicted to accumulate during cell division. ${ }^{2}$ Microsatellite instability (MSI) has received attention since the discovery of repetitive sequences in 1993. ${ }^{3}$ Deficient mismatch repair (dMMR) is the molecular basis for MSI. Two distinct causal mechanisms have been identified to explain these deficiencies. One potential mechanism is germline mutations in MLH1, MSH2, MSH6, and PMS2. Approximately $3 \%$ of CRC cases are associated with germline mutations in MMR genes. ${ }^{4}$ Lynch syndrome is caused by these germline mutations in MMR genes; it is associated with extraintestinal manifestations, including cancers in the endometrium, ovary, stomach, hepatobiliary tract, and urinary tract. ${ }^{5}$ The other mechanism is related to the methylation of $\mathrm{CpG}$ (cytosinephosphate-guanine) islands of MLH1., ${ }^{1,5}$ This MSI phenotype holds clinical importance, as it provides predictive value with respect to prognosis and response to chemotherapy. ${ }^{6}$
Many groups have examined the correlation in MSI status between primary and secondary CRCs. In many cases, MSI is found in the primary tumor, but not in the metastatic lesion, suggesting that MMR deficiencies contribute to tumor initiation, rather than progression. ${ }^{2}$ Although MSI groups show favorable outcomes compared to the microsatellite stable groups in primary CRCs, little is known about the frequency of MSI and its importance in secondary CRCs.

The current gold standard for assessing DNA MMR competency is polymerase chain reaction (PCR). ${ }^{7}$ However, immunohistochemical staining (IHC) for DNA MMR proteins (e.g., MLH1, MSH2, MSH6, and PMS2) has emerged as a useful complementary technique. ${ }^{8}$ Compared to PCR, the sensitivity of IHC is as high as $93 \%$ and the specificity is close to perfect. ${ }^{5,9,10}$

MMR proteins recognize and correct insertion/deletion loops, base mismatches, and damaged bases. ${ }^{11}$ They can interact as heterodimeric complexes: MSH2-MSH6, MSH2-MSH3, MLH1PMS2, MLH1-PMS1, or MLH1-MSH3 ${ }^{6}$ In 1996, the expression 
of the MSH2 antibody was first reported and developed as a marker using fresh-frozen tissues. ${ }^{12}$ Later, antibodies against MLH1 and MSH2 became applicable to paraffin embedded tissues. ${ }^{13}$ Since then, numerous MMR IHC studies have been performed.

Shia et al. ${ }^{10}$ showed that the overall sensitivity of IHC for the prediction of germline mutations is $79 \%$, with a specificity of $89 \%$. In another study, IHC correctly predicted the MSI status in $76 \%$ of cases, with a specificity of $100 \%$.

The aim of this study was to compare the MMR system between primary CRCs and paired liver and lung metastatic lesions using IHC and to determine whether there is a correlation with the clinical outcomes.

\section{MATERIALS AND METHODS}

\section{Patient population}

The database of the Korea University Anam Hospital, Seoul, Korea was searched for all CRC patients who underwent surgical resection between 2002 and 2013. A cohort of patients who underwent hepatic resection or pulmonary resection for CRC metastases was also identified. Patients with available tissue samples were analyzed for MMR using IHC. Patients who received neoadjuvant chemoradiotherapy were excluded. A total of 61 cases were included in the study. The corresponding clinical data were obtained from a retrospective review of patient records. Follow-up survival data were also obtained.

\section{Immunohistochemistry}

To characterize the MMR system, IHC was performed using $4-\mu \mathrm{m}$-thick paraffin tissue sections. The manufacturers and incubation conditions for primary antibodies are summarized in Table 1. Sections were incubated for 15 minutes with antibodies against MLH1 protein (1:200, ES05, Leica, Newcastle upon Tyne, UK), MSH2 protein (1:100, G219-1129, Cell Marque, Rocklin, CA, USA), MSH6 protein (1:50, 44, Cell Marque), and PMS2 (1:50, MRQ-28, Cell Marque).

Tumors were considered deficient in MLH1, MSH2, MSH6, and PMS2 expression when there was a complete absence of detectable nuclear staining in neoplastic cells. Intact nuclear staining of the adjacent non-neoplastic epithelium, stromal cells, or lymphocytes served as an internal positive control (Appendix 1).

Whole sections were stained and reviewed for cases that showed a loss of expression on a microarray.

\section{Statistical analysis}

Clinicopathological features, including age, size, gender, site of primary CRC, lymph node status, lymphovascular invasion, perineural invasion, carcinoembryonic antigen (CEA) levels at diagnosis, $\mathrm{T}$ category, tumor differentiation, and metastatic site, were compared between dMMR and proficient MMR (pMMR) patients used Fisher exact, $\chi^{2}$ tests and Mann-Whitney test. Overall survival was calculated according to the Kaplan-Meier method. All statistical tests were implemented in SPSS ver. 21 (IBM Corp., Armonk, NY, USA).

This case was reviewed by the Institutional Review Board of Korea University Medical Center (AN15349-00).

\section{RESULTS}

\section{Clinicopathological features}

The clinicopathological features of 61 patients included in the study are detailed in Table 1 . The mean patient age was 58 years (range, 31 to 78 years); $26 \%$ of the patients were female and $73 \%$ were male. The mean tumor size was $5.4 \mathrm{~cm}$ (range, 1.8 to $15 \mathrm{~cm}$ ). The anatomical location of the primary tumor was classified as left (rectum, rectosigmoid colon, splenic flexure, and descending colon) or right side (ascending colon, hepatic flexure, and cecum). In total, 55/61 (90.1\%) were T3 cancers that invade through the muscularis propria into pericolorectal tissues. Moderately differentiated CRCs accounted for 44/61 (72.1\%).

There were two patients in their early thirties. Neither patient had a family history of cancer.

\section{Relationship between MMR status and clinicopathological features}

Using Fisher exact, $\chi^{2}$ tests and Mann-Whitney tests to analyze the relationship between MMR status and clinicopathological features, significant relationships were not detected for age $(\mathrm{p}=$ .58), size $(\mathrm{p}=.14)$, sex $(\mathrm{p}=.34)$, site $(\mathrm{p}=.96)$, T stage $(\mathrm{p}=.36)$, tumor differentiation $(\mathrm{p}=.32)$ lymph node metastasis $(\mathrm{p}=.13)$, lymphovascular invasion $(\mathrm{p}=.75)$, perineural invasion $(\mathrm{p}=.30)$, or CEA level $(\mathrm{p}=.49)$.

\section{Association between primary and metastatic tumors}

A total of 61 CRCs were assessed using a tissue microarray. The results are detailed in Tables 2 and 3 .

Of the primary tumors, $7 / 61$ (11.4\%) showed a dMMR system based on IHC, while secondary tumors showed a deficiency in 13/61 cases (21.3\%). In total, 44 cases showed proficient expression of MMR in both primary and metastatic lesions. Three cases 
Table 1. Clinicopathological features of the entire cohort

\begin{tabular}{|c|c|c|c|c|}
\hline Variable & MMR-proficient $(n=44)$ & MMR-deficient $(n=17)$ & $\chi^{2}$ & $\mathrm{p}$-value \\
\hline Age at diagnosis (yr) & $54(31-78)$ & $55(41-74)$ & - & $.58^{\mathrm{a}}$ \\
\hline Size $(\mathrm{cm})$ & $5.1(1.8-11.0)$ & $6.1(1.8-15.0)$ & - & $.14^{\mathrm{a}}$ \\
\hline Sex & & & 0.897 & $.34^{\mathrm{b}}$ \\
\hline Male & 31 & 14 & & \\
\hline Female & 13 & 3 & & \\
\hline Anatomic location & & & 0.002 & $.96^{b}$ \\
\hline Right & 8 & 3 & & \\
\hline Left & 36 & 14 & & \\
\hline T stage & & & 2.074 & $.36^{b}$ \\
\hline $\mathrm{T} 1$ & 0 & 0 & & \\
\hline T2 & 4 & 0 & & \\
\hline T3 & 39 & 16 & & \\
\hline T4 & 1 & 1 & & \\
\hline Differentiation & & & 3.498 & $.32^{\mathrm{b}}$ \\
\hline Well & 12 & 3 & & \\
\hline Moderate & 31 & 13 & & \\
\hline Poor & 1 & 0 & & \\
\hline Mucinous & 0 & 1 & & \\
\hline Lymph node metastasis & & & 2.273 & $.13^{\mathrm{b}}$ \\
\hline No & 19 & 11 & & \\
\hline Yes & 25 & 6 & & \\
\hline Lymphovascular space invasion & & & 0.123 & $.75^{\mathrm{b}}$ \\
\hline No & 33 & 12 & & \\
\hline Yes & 11 & 5 & & \\
\hline Perineural invasion & & & 1.082 & $.30^{\mathrm{b}}$ \\
\hline No & 37 & 16 & & \\
\hline Yes & 7 & 1 & & \\
\hline CEA at diagnosis (ng/mL) & $37.1(0.2-1,042.8)$ & $63.4(1.1-852.5)$ & - & $.49^{\mathrm{a}}$ \\
\hline \multicolumn{5}{|l|}{ Metastastic site } \\
\hline Liver & 21 & 15 & & \\
\hline Lung & 23 & 2 & & \\
\hline
\end{tabular}

Values are presented as mean (range) or number.

MMR, mismatch repair; CEA, carcinoembryonic antigen.

${ }^{a}$ Mann-Whitney test; ${ }^{b}$ Fisher exact or $\chi^{2}$ tests.

Table 2. MMR status based on the $\mathrm{HC}$ analysis for primary and metastatic tumors

\begin{tabular}{|c|c|c|c|}
\hline \multirow{2}{*}{ Metastasis } & \multicolumn{2}{|c|}{ Primary } & \multirow{2}{*}{ Total } \\
\hline & Intact & Loss & \\
\hline Intact & 44 & 4 & 48 \\
\hline Loss & 10 & 3 & 13 \\
\hline Total & 54 & 7 & \\
\hline
\end{tabular}

MMR, mismatch repair; $1 \mathrm{HC}$, immunohistochemical staining.

showed deficiencies in both the primary and the paired metastatic lesions. In 10 cases, proficient expression was found in the primary lesions, but not in the corresponding metastatic lesions. In four cases, proficient expression was detected in the secondary tumor, but not in the primary tumor.
Table 3. Immunohistochemical patterns of mismatch repair deficiencies

\begin{tabular}{lcc}
\hline \multirow{2}{*}{ Loss } & \multicolumn{2}{c}{ Tumor } \\
\cline { 2 - 3 } & Primary & Metastatic \\
\hline MLH1/PMS2 & 0 & 0 \\
MSH2/MSH6 & 6 & 8 \\
MSH6 only & 0 & 2 \\
PMS2 only & 1 & 1 \\
Combined $^{a}$ & 0 & 3 \\
\hline
\end{tabular}

${ }^{a}$ Combined: MSH6 + PMS2 (1 case), MLH1 + MSH6 + PMS2 (1 case), MSH2 + PMS2 (1 case).

\section{Survival analysis}

In total, 14 patients died of cancer, and the median survival was 33 months from the date of initial diagnosis.

A survival analysis was performed assuming that the primary and metastatic tumors showing intact expression using IHC 


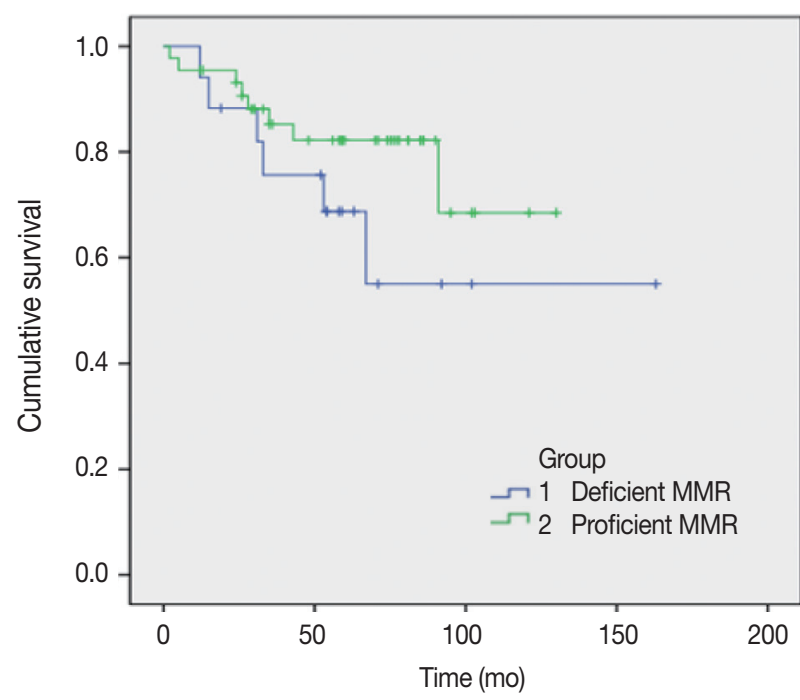

Fig. 1. Overall survival for the entire cohort (proficient mismatch repair [MMR] and deficient MMR patients).

group are pMMR and the loss of expression in either tumor indicates dMMR (Fig. 1). This result was not statistically significant (log-rank test, $\mathrm{p}=.22$ ), but the pMMR group showed a more favorable prognosis compared to the dMMR group.

\section{DISCUSSION}

In this study, MMR proteins were evaluated by IHC for the following reasons: (1) IHC is less time consuming than PCR, (2) is easier to implement, ${ }^{14}$ and (3) enables the simultaneous identification of affected MMR genes. ${ }^{5}$

However, the interpretation of IHC results requires caution for the following reasons. First, it is well known that the IHC staining patterns for MSH6 IHC are variable. ${ }^{8}$ Second, stainability is dependent on tissue handling, including fixation and analytical variables. For instance, tissue hypoxia and delayed fixation reduce the sensitivity of detection of MMR gene expression or suppress MMR. Fewer proliferative cells result in lower levels of staining. ${ }^{15}$ Third, some MLH1 mutation-positive cases or even cases with $M L H 1$ promoter methylation show false-positive nuclear staining. ${ }^{6,9} \mathrm{~A}$ missense mutation or an in-frame insertion/deletion mutation in MLH1 does not affect MLH1-PMS2 interactions, and thus the protein reacts with the antibody used for IHC. Lastly, interpretation of MMR IHC results requires consideration that the MMR proteins act as heterodimers. ${ }^{6}$ For example, CRCs that show loss of MLH1 and PMS2 expression, but intact MSH2 and MSH6 expression indicate an MLH1 deficiency. The loss of PMS2 expression results from $\mathrm{MLH1}$ mutations.

In this study, our major interest was investigating the con- cordance in MMR deficiencies between the primary CRCs and its corresponding metastases.

Haraldsdottir et al. ${ }^{16}$ reported matching IHC results between the primary tumor and metastatic tissue for all cases examined. However, Agoston et al. ${ }^{17}$ found discordance in the MMR status between primary tumors and metastases in $20.2 \%$ of cases. Haddad $e t$ al. ${ }^{18}$ found a very low frequency of MSI using PCR in resected CRC hepatic metastases in 190 patients. Unlike in primary CRCs, the rate of MSI in resectable CRC hepatic metastases is approximately $2.5 \%$. Two potential explanations were proposed to explain this discrepancy. First, high-frequency MSI primary CRCs do not frequently metastasize to the liver. Second, high-frequency MSI primary CRCs spread to the liver extensively and therefore are considered unresectable. ${ }^{18}$ Previous studies suggest divergent views regarding this issue.

Of the 61 cases, 47 showed concordance in the IHC staining results between the primary and metastatic tumors. The incidence of dMMR was similar to the previously reported incidence of $10 \%-20 \% .{ }^{19}$ However, the incidence was somewhat different between the primary (11.5\%) and the metastatic (21.3\%) cancer groups. In this study, among the 36 hepatic metastatic cancers, five cases showed dMMR in primary CRCs. This frequency of approximately $14 \%$ was not as low as that reported in the previous study.

We focused on dMMR cases that showed discordance between primary and metastatic tumors. The clinicopathological features and each MMR protein expressions of the cases with either primary or metastatic dMMR are summarized in Tables 4 and 5. Except one case, 16 cases were T3 cancers. Most of the cases $(16 / 17)$ presented without perineural invasion. Based on IHC, primary and metastatic lesions of all the cases showed intact MLH1 expression. In 10 cases, proficient expression was found in the primary tumor, but not in the corresponding metastatic lesions. In four cases, proficient expression was detected in the secondary tumor, but not in the primary tumor. Furthermore, the precise MMR proteins that showed a loss of expression were not exactly matched in the primary and the metastatic tumors.

To further examine the data, we divided the dMMR cases into five groups. During heterodimeric complex formation, MMR proteins change concurrently. The loss of PMS2 is followed by the loss of MLH1 expression owing to functional dimerization. Similarly, the loss of MSH2 is accompanied by the loss of MSH6 (Fig. 2). However, the loss of isolated MSH6 or PMS2 is not accompanied by MLH1 or MSH2, reflecting mutations in MSH6 or PMS2. Although three cases were classified as a combined group that do not belong to the above four groups, most 
Table 4. Clinicopathological features of dMMR cases

\begin{tabular}{|c|c|c|c|c|c|c|c|c|c|c|c|c|}
\hline Case & Sex & Age (yr) & Location & Size & T stage & LN metastases & LVI & $\mathrm{PNI}$ & CEA (ng/mL) & Survival time (mo) & Evolution & Metastasis \\
\hline$\overline{1}$ & $M$ & 62 & $\mathrm{R}$ & 5.2 & III & + & + & - & 2.8 & 31 & $\mathrm{D}$ & Liver \\
\hline 2 & $\mathrm{~F}$ & 46 & $\mathrm{R}$ & 2.5 & IV & - & - & - & 2.9 & 163 & A & Liver \\
\hline 3 & M & 61 & $\mathrm{R}$ & 5 & III & - & - & - & 3.3 & 102 & A & Liver \\
\hline 4 & M & 49 & $\mathrm{RS}$ & 5 & III & + & + & - & 7 & 53 & D & Liver \\
\hline 5 & M & 74 & $S$ & 8.8 & III & - & - & - & 1.2 & 54 & A & Liver \\
\hline 6 & $\mathrm{~F}$ & 67 & S & 1.8 & III & - & + & + & 3.6 & 71 & A & Liver \\
\hline 7 & M & 53 & $S$ & 7 & III & - & - & - & 7.8 & 59 & A & Liver \\
\hline 8 & $\mathrm{~F}$ & 41 & As & 15 & III & - & - & - & 4.4 & 15 & D & Liver \\
\hline 9 & $M$ & 58 & $S$ & 3.5 & III & + & - & - & 2.7 & 58 & A & Liver \\
\hline 10 & $M$ & 57 & $\mathrm{Hf}$ & 7.5 & III & - & - & - & 852.5 & 54 & A & Liver \\
\hline 11 & $M$ & 57 & $\mathrm{RS}$ & 6 & III & - & - & - & 124.5 & 63 & A & Liver \\
\hline 12 & $M$ & 64 & $S$ & 6.3 & III & + & - & - & 2.22 & 92 & A & Liver \\
\hline 13 & $M$ & 59 & As & 3 & III & - & + & - & 1.1 & 33 & $D$ & Liver \\
\hline 14 & $M$ & 67 & $\mathrm{R}$ & 5.5 & III & + & - & - & 11.4 & 12 & $D$ & Liver \\
\hline 15 & $M$ & 54 & $S$ & 7.5 & III & - & - & - & 2 & 19 & A & Liver \\
\hline 16 & $M$ & 64 & $\mathrm{R}$ & 6.5 & III & + & - & - & 13.7 & 67 & D & Lung \\
\hline 17 & $M$ & 68 & Sf & 8 & III & - & + & - & 34.3 & 52 & A & Lung \\
\hline
\end{tabular}

dMMR, deficient mismatch repair; LN, lymph node; LVI, Iymphovascular invasion; PNI, perineural invasion; CEA, carcinoembryonic antigen; M, male; R, rectum; +, present; -, absent; D, death; F, female; A, alive; RS, rectosigmoid colon; S, sigmoid colon; As, ascending colon; Hf, hepatic flexure; Sf, splenic flexure.

Table 5. MMR protein expression status of primary and metastatic colorectal adenocarcinomas in dMMR cases

\begin{tabular}{|c|c|c|c|c|c|}
\hline Case & MLH1 (P/M) & MSH2 (P/M) & MSH6 (P/M) & PMS2 (P/M) & MMR (P/M) \\
\hline 1 & I/I & I/I & I/L & I/L & $\mathrm{Pr} / \mathrm{De}$ \\
\hline 2 & I/I & L/I & L/I & I/I & $\mathrm{De} / \mathrm{Pr}$ \\
\hline 3 & I/I & I/I & $1 / L$ & 1// & $\mathrm{Pr} / \mathrm{De}$ \\
\hline 4 & $1 / 1$ & $1 / L$ & $1 / L$ & |/I & $\mathrm{Pr} / \mathrm{De}$ \\
\hline 5 & I/I & $1 / L$ & I/I & L/I & De/De \\
\hline 6 & 1/I & L/I & $1 / /$ & $1 / /$ & $\mathrm{De} / \mathrm{Pr}$ \\
\hline 7 & I/I & $1 / /$ & I/I & $1 / L$ & $\mathrm{Pr} / \mathrm{De}$ \\
\hline 8 & 1/I & $\mathrm{L} / \mathrm{L}$ & $\mathrm{L} / \mathrm{L}$ & $1 / 1$ & $\mathrm{De} / \mathrm{De}$ \\
\hline 9 & l/I & $\mathrm{I} / \mathrm{L}$ & $\mathrm{I} / \mathrm{L}$ & 1// & $\mathrm{Pr} / \mathrm{De}$ \\
\hline 10 & I/L & $1 / 1$ & $1 / L$ & $1 / /$ & $\mathrm{Pr} / \mathrm{De}$ \\
\hline 11 & l/I & $\mathrm{l} / \mathrm{L}$ & $1 / L$ & l/I & Pr/De \\
\hline 12 & I/I & $\mathrm{L} / \mathrm{L}$ & $1 / L$ & $1 / L$ & De/De \\
\hline 13 & I/I & $\mathrm{I} / \mathrm{L}$ & I/I & I/I & Pr/De \\
\hline 14 & I/I & $1 / L$ & 1/I & 1/I & Pr/De \\
\hline 15 & I/l & $\mathrm{I} / \mathrm{L}$ & $1 / L$ & I/I & $\mathrm{Pr} / \mathrm{De}$ \\
\hline 16 & I/I & L/I & L/I & $1 / 1$ & $\mathrm{De} / \mathrm{Pr}$ \\
\hline 17 & $1 / 1$ & $\mathrm{~L} / \mathrm{I}$ & L/I & $1 / 1$ & $\mathrm{De} / \mathrm{Pr}$ \\
\hline
\end{tabular}

P, primary; M, netastatic; MMR, mismatch repair; I, intact; L, loss; Pr, proficient; De, deficient.

of the dMMR cases fit the classification, indicating that the IHC expression is consistent.

MLH1 mutations are the most common type among the MMR genes. However, the detected loss of the MLH1 protein was significantly less frequent than the loss of the MSH2 protein in this study. As stated above, we attributed this difference to the IHC technique. Most mutations in MSH2 result in truncated proteins, which consequently showed a loss of expression in IHC analyses. However, MLH1 mutations are non-functional missense mutations, and mutated-MLH1 cases also show profi- cient expression. ${ }^{15}$ A meta-analysis showed that using IHC, only $74 \%$ of MLH1 losses were detected in the MLH1 mutationpositive cases, compared to the $91 \%$ detected using MSI testing. However, MSH2 mutations could be detected in up to $94 \%$ of the MSH2 mutation-positive cases by IHC. ${ }^{9}$ We infer that there may be false-positive MLH1 cases, and additional PCR studies are needed to identify the precise $M L H 1$ mutations.

In our study, cases were considered deficient if any target locus (e.g., MLH1, MSH2, MSH6, or PMS2) was not expressed. This is a limitation of our study, and variable patterns could lead to 
erroneous interpretations. Shia et al. ${ }^{8}$ analyzed MSH6 using IHC and showed limited staining in the MLH1/PMS2-deficient patients. In one case, we detected the losses of MLH1, MSH6, and PMS2 expression, suggesting a similar scenario.

Some cases showed a loss of MSH2 expression, but intact MSH6 expression. We attributed this discordance in IHC results to the variable reactivity and subjective data interpretations. Further evaluation by PCR is needed to obtain more definitive results.

All cases received adjuvant chemotherapy after resection of the primary tumor, because all cases included in this study were stage IV. Most of the patients (56 cases) were treated with FOLFOX regimens (combination of folic acid, 5-fluorouracil
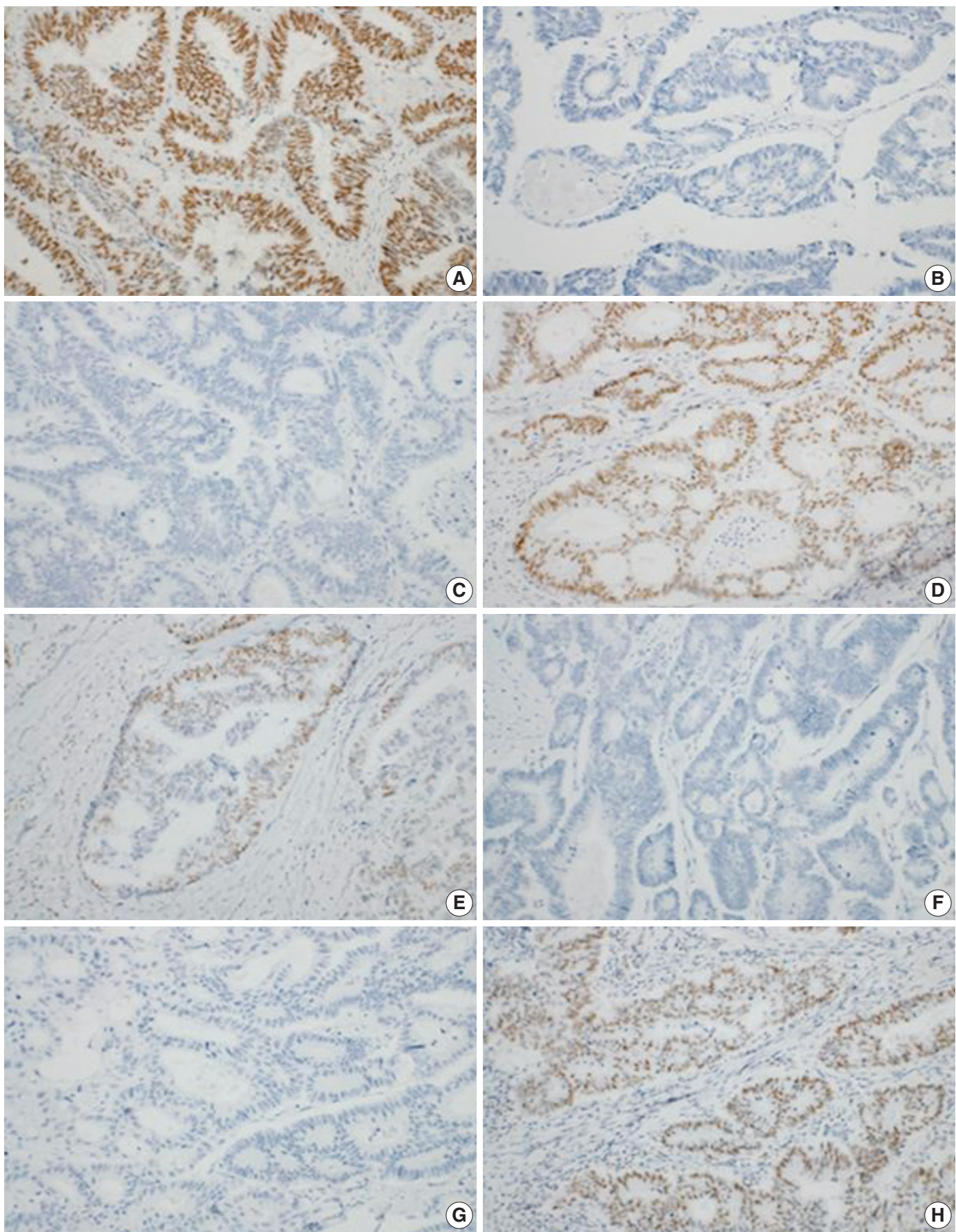

Fig. 2. Immunohistochemical analyses of primary colorectal cancer for MLH1 (A), MSH2 (B), MSH6 (C), and PMS2 (D). Immunohistochemical analyses of metastatic colorectal cancer for MLH1 (E), MSH2 (F), MSH6 (G), and PMS2 (H). A lack of staining for MSH2 and MSH6 indicates a primary defect in $\mathrm{MSH} 2$, with a secondary loss of MSH6 expression. 
[5-FU] and oxaliplatin). Two cases were treated with leucovorin and 5-FU without oxaliplatin. Three cases were treated only with 5-FU-based chemotherapy. Among the 61 cases, 50 cases received chemotherapy before metastatectomy, wheres 11 cases received chemotherapy after metastatectomy. Of the 50 cases that received chemotherapy before metastatectomy, 11 cases (22\%) showed discordance in IHC. In seven cases, proficient expression was found in the primary lesions, but not in the corresponding metastatic lesions. We assume that these losses of expression could be associated with chemotherapy-related effect.

MMR status was not a significant predictive marker in metastatic CRCs. This finding concurs with the results of previous studies..$^{20}$ Since all cases were stage IV tumors, the low survival rates were expected.

Despite these limitations, we detected a correlation between the MMR status of primary and metastatic CRCs using only IHC. These findings may improve our understanding of the metastatic processes in CRC patients. Further research should focus on the modifications in the biological and biochemical properties of DNA MMR proteins during metastatic processes.

\section{Conflicts of Interest}

No potential conflict of interest relevant to this article was reported.

\section{REFERENCES}

1. Kanthan R, Senger JL, Kanthan SC. Molecular events in primary and metastatic colorectal carcinoma: a review. Patholog Res Int 2012; 2012: 597497.

2. Yim KL. Microsatellite instability in metastatic colorectal cancer: a review of pathology, response to chemotherapy and clinical outcome. Med Oncol 2012; 29: 1796-801.

3. Aaltonen LA, Peltomäki P, Leach FS, et al. Clues to the pathogenesis of familial colorectal cancer. Science 1993; 260: 812-6.

4. Geiersbach KB, Samowitz WS. Microsatellite instability and colorectal cancer. Arch Pathol Lab Med 2011; 135: 1269-77.

5. Shia J. Evolving approach and clinical significance of detecting DNA mismatch repair deficiency in colorectal carcinoma. Semin Diagn Pathol 2015; 32: 352-61.

6. Vilar E, Gruber SB. Microsatellite instability in colorectal cancer: the stable evidence. Nat Rev Clin Oncol 2010; 7: 153-62.

7. Chen S, Watson P, Parmigiani G. Accuracy of MSI testing in predicting germline mutations of MSH2 and MLH1: a case study in Bayesian meta-analysis of diagnostic tests without a gold standard.
Biostatistics 2005; 6: 450-64.

8. Shia J, Zhang L, Shike M, et al. Secondary mutation in a coding mononucleotide tract in MSH6 causes loss of immunoexpression of MSH6 in colorectal carcinomas with MLH1/PMS2 deficiency. Mod Pathol 2013; 26: 131-8.

9. Zhang L. Immunohistochemistry versus microsatellite instability testing for screening colorectal cancer patients at risk for hereditary nonpolyposis colorectal cancer syndrome. Part II. The utility of microsatellite instability testing. J Mol Diagn 2008; 10: 301-7.

10. Shia J, Klimstra DS, Nafa K, et al. Value of immunohistochemical detection of DNA mismatch repair proteins in predicting germline mutation in hereditary colorectal neoplasms. Am J Surg Pathol 2005; 29: 96-104.

11. Poulogiannis G, Frayling IM, Arends MJ. DNA mismatch repair deficiency in sporadic colorectal cancer and Lynch syndrome. Histopathology 2010; 56: 167-79.

12. Leach FS, Polyak K, Burrell M, et al. Expression of the human mismatch repair gene hMSH2 in normal and neoplastic tissues. Cancer Res 1996; 56: 235-40.

13. Thibodeau SN, French AJ, Roche PC, et al. Altered expression of hMSH2 and hMLH1 in tumors with microsatellite instability and genetic alterations in mismatch repair genes. Cancer Res 1996; 56: 4836-40.

14. Lindor NM, Burgart LJ, Leontovich O, et al. Immunohistochemistry versus microsatellite instability testing in phenotyping colorectal tumors. J Clin Oncol 2002; 20: 1043-8.

15. Shia J, Holck S, Depetris G, Greenson JK, Klimstra DS. Lynch syndrome-associated neoplasms: a discussion on histopathology and immunohistochemistry. Fam Cancer 2013; 12: 241-60.

16. Haraldsdottir S, Roth R, Pearlman R, Hampel H, Arnold CA, Frankel WL. Mismatch repair deficiency concordance between primary colorectal cancer and corresponding metastasis. Fam Cancer 2016; 15: 253-60.

17. Agoston EI, Baranyai Z, Dede K, et al. Occurrence, intratumoral heterogeneity, prognostic and predictive potential of microsatellite instability following surgical resection of primary colorectal carcinomas and corresponding liver metastases. Orv Hetil 2015; 156: 1460-71.

18. Haddad R, Ogilvie RT, Croitoru M, et al. Microsatellite instability as a prognostic factor in resected colorectal cancer liver metastases. Ann Surg Oncol 2004; 11: 977-82.

19. Koopman M, Kortman GA, Mekenkamp L, et al. Deficient mismatch repair system in patients with sporadic advanced colorectal cancer. Br J Cancer 2009; 100: 266-73.

20. Kim ST, Lee J, Park SH, et al. The effect of DNA mismatch repair (MMR) status on oxaliplatin-based first-line chemotherapy as in recurrent or metastatic colon cancer. Med Oncol 2010; 27: 1277-85. 


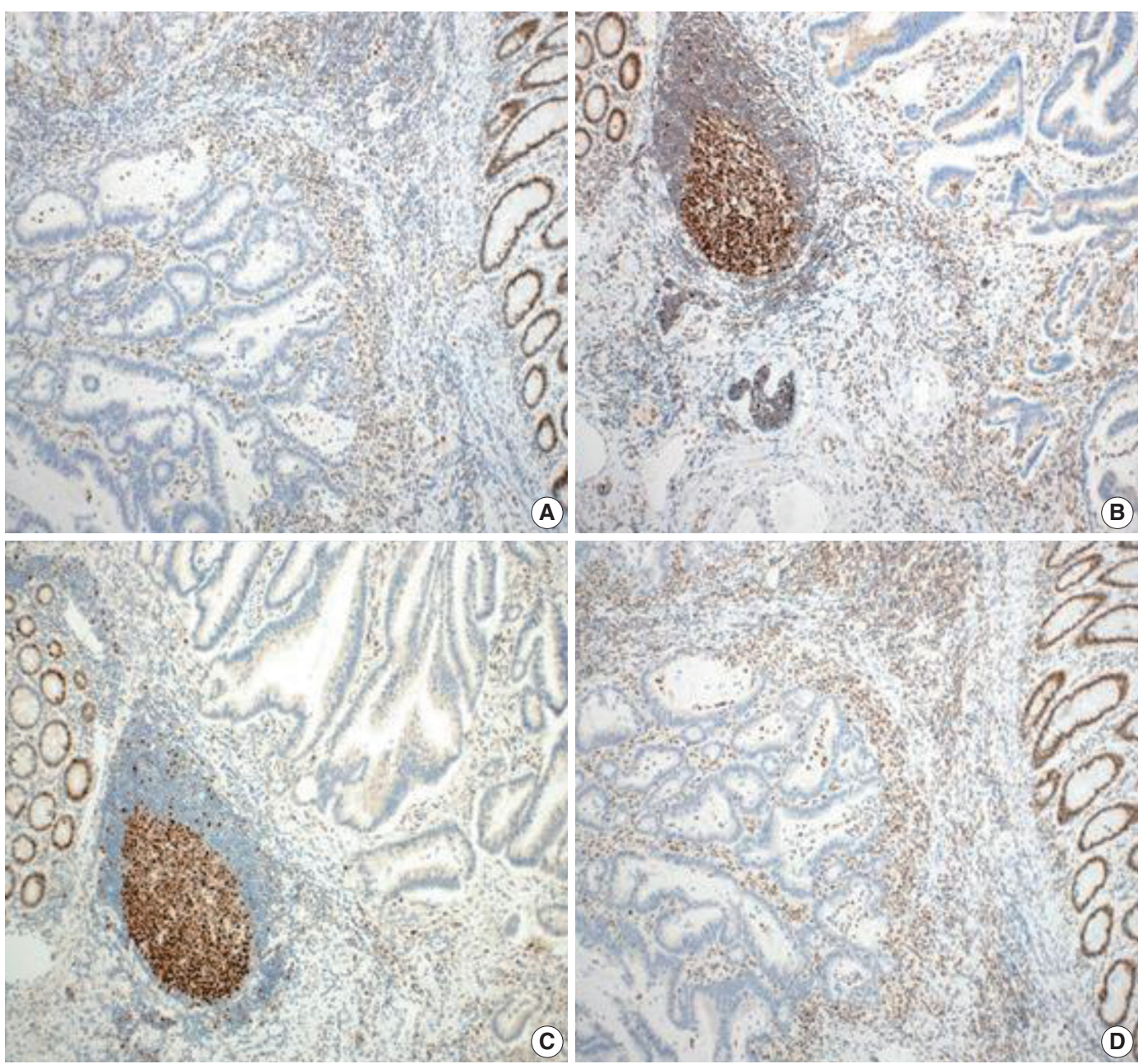

Appendix 1. Absence of immunohistochemical staining in tumor epithelium for MLH1 (A), MSH2 (B), MSH6 (C), and PMS2 (D). Note the presence of tumor-infiltrating lymphocytes and adjacent normal mucosal tissue that stain positively for each immunohistochemical staining. 